\title{
Despeckling of Ultrasound Medical Images using DW and WP Transform Techniques
}

\author{
P. Kalavathi ${ }^{\# 1}$, S. Boopathiraja ${ }^{\# 2}$ and M. Abinaya ${ }^{\# 3}$ \\ \#Department of Computer Science and Applications \\ The Gandhigram Rural Institute-Deemed University, Gandhigram - 624 302, Tamilnadu, India \\ pkalavathi.gri@gmail.com \\ 2boopathiraja777@gmail.com \\ 3abinayasree91@gmail.com
}

\begin{abstract}
The existences of noise in the medical images impose misleading of diagnostic processes, because of the radiation problem while acquiring images. The medical images mostly affected by the speckle noise over other natural noises. There are number of noise removal techniques for speckle noise is proposed in both spatial and frequency domain. In this paper, we propose a frequency domain method using wavelet based noise removal technique to remove speckle noise that exists in the ultrasound images. Moreover, we have implemented two different methods in terms of decomposing the images. We used Discrete Wavelet Transform (DWT) and Wavelet Packet Transform (WPT) for decompose the images. For comparative analysis, the performance of these filtering techniques is quantitatively evaluated through Peak Signal to Noise Ratio (PSNR).
\end{abstract}

Keyword-Speckle Noise, Ultrasound Image, Discrete Wavelet Transform (DWT), Wavelet Packet Transform (WPT).

\section{INTRODUCTION}

In the medical imaging field, the noises occurring in the images are a crucial issue that leads to faulty decision during the patient diagnostics processes. There are variety of noise removal techniques also exists for mixed noises [1]. Noise removal techniques are constructed through spatial or frequency domain. Spatial filtering is nothing but the filtering operations that are performed directly on the intensity of the pixels [2]. It works by simply moving the kernel (i.e. filter mask) throughout the image from point to point. It mainly classified into smoothing, order-statistics and sharpening filtering. Ultrasound is one of the low cost medical imaging modality with simpler operations and so that used widely. High frequency sound waves are used to capture the inner organs [3]. The ultrasound image is produced by the reflection of the waves from the body structures. The amplitude of the signal and time taken for the wave to travel through the body are the two main factors to construct an ultrasound image. Moreover, ultrasound is a sound wave with frequencies higher than the upper audible limit of human hearing. The frequencies that operated in ultrasound devices are ranges from $20 \mathrm{kHz}$ up to several gigahertz. Health care professionals uses ultrasound images to view the internal organs. During pregnancy, doctors are uses ultrasound to view the fetus, because it does not expose any radiation like other modalities. The noise occurrence in this modality is a big problem that occurs due to the value of the clear nature of the trend transferring [4]. These noises corrupt the images and often lead to incorrect diagnosis [5]. Medical imaging modalities are affected by different types of noises especially the ultrasound images are mainly affected by speckle noise.

Speckle is a granular noise that exists inherently and it degrades the quality of the images such as synthetic aperture radar (SAR), medical ultrasound and optical coherence tomography images [6]. Reducing these speckle noise from a noisy image is the complicated step in medical image processing. Speckle noise degrades image quality with a backscattered wave appearance which originates from many sources like microscopic spreader reflections that pass through internal organs. It makes much difficulty for the observer to discriminate fine detail of the images while diagnostic processes. Thus, denoising or reducing these speckle noise from a noisy image has become the predominant step in medical field. Some spatial domain filtering techniques are existing for removing noises [7]. Ultimately, the speckle noise addresses the contrast related problems on images. So, there is great need to construct a denoising method to remove the speckle [8]. In this paper, we experimented the ultrasound images which are affected by speckle noise for denoising. A wavelet based image filtering technique is applied to ultrasound images which effectively remove the noises. In addition, the performance of aforementioned filters is compared (in terms of PSNR) and discussed in the following sections. This paper is further organized as follows: Section 2 contains the brief introduction about the Discrete Wavelet Transform (DWT) and Wavelet Packet Transform (WPT), our proposed method is explained in section 3, the results and discussion included in section 4 and we conclude our paper in section 5. 


\section{Discrete Wavelet Transform AND WAVElet PaCKet Transform}

A multiresolution analysis formally introduced to image processing by Mallat [9]. It provides a way for simultaneous analyzing of digital images in different scales. In practical, an image is analysed in different frequencies with various resolution. The Discrete Wavelet Transform (DWT) encompasses the properties of multiresolution analysis which analyze the images progressively into finer sub-bands. In digital images, wavelets have done the scaling and translation through morphological operations. An image is applied with two filters namely high-pass and low-pass filters and it yields one approximation sub-band and three detail coefficients [10]. These operations are progressively applied on approximation sub-band of next successive decomposition levels. The Fig. 1 shows the decomposition structures of 2D-DWT.

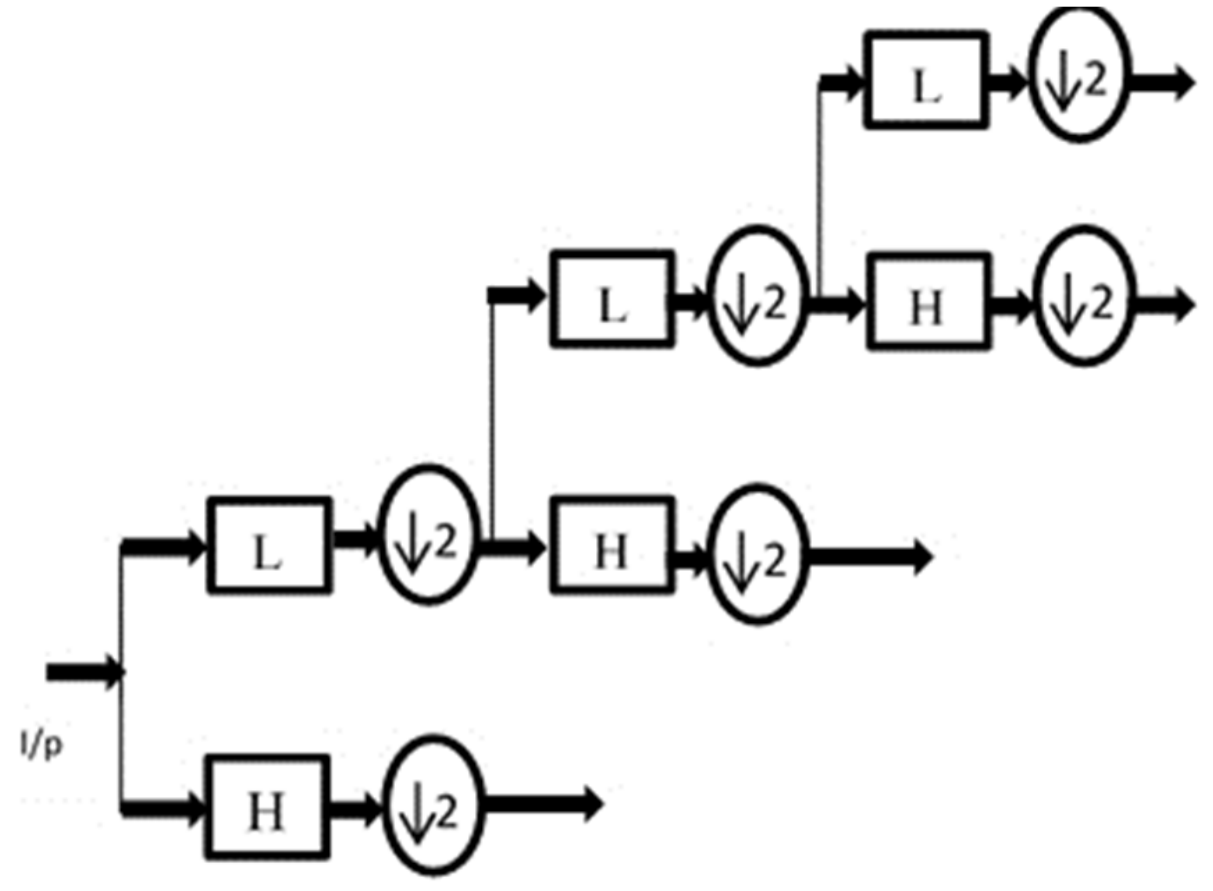

Fig1.Decomposition structure of DWT

The generalization of wavelet decomposition structure is known as the wavelet packet transforms (WPT) [11]. In the way of applying filters on an image, it differs from the DWT. Approximation details only decomposed further (applying complementary filters) in DWT but the detail sub-bands also decomposed in WPT which is depicted in Fig.2. The optimal decomposition tree is then chosen as final decomposition structure based on the entropy-based criterion for the given level [12].

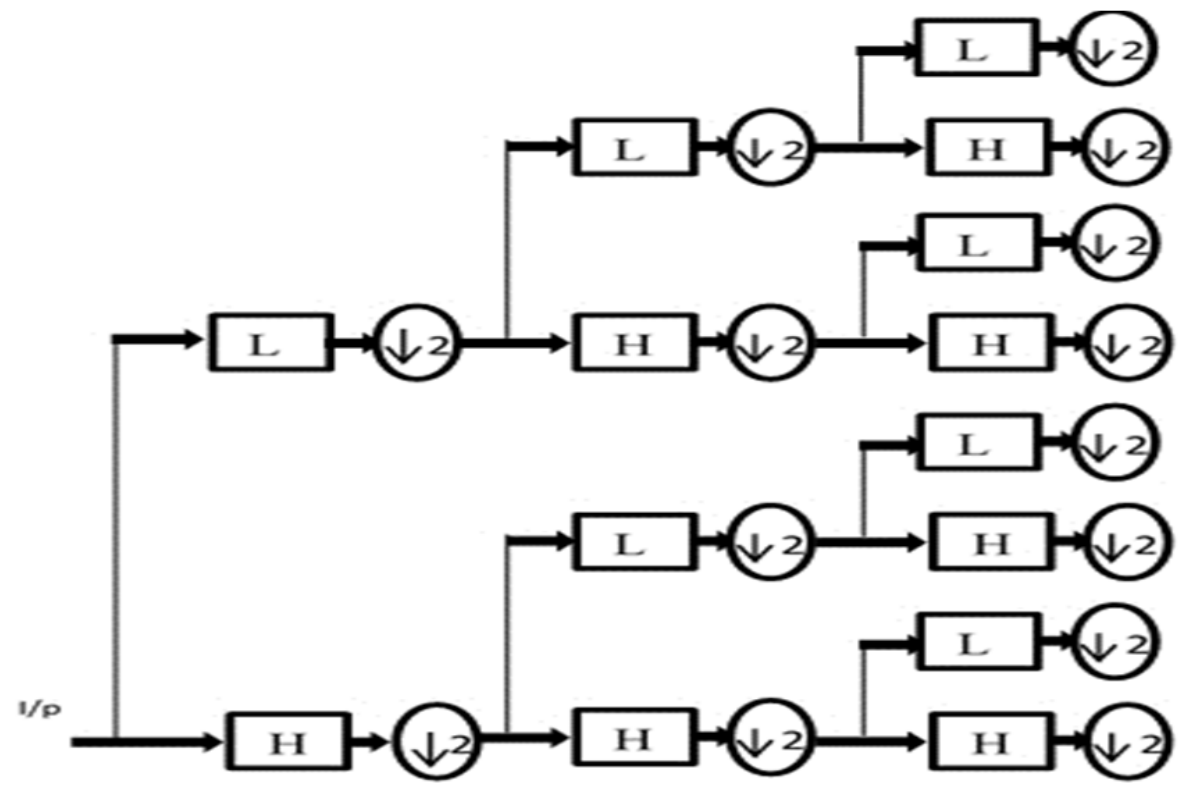

Fig2. Decomposition structure of WPT 


\section{III.PROPOSED METHOD}

\section{A. Methodology}

As we proposed a wavelet based method, a noisy image is decomposed with a wavelet basis. In this paper, we used two different wavelet decomposition structures such as Discrete Wavelet Transform (DWT) and Wavelet Packet Transform (WPT). The results of using these two methods are depicted and the comparison of those two strategies is discussed separately in following sections. The implementation steps of our proposed method are given as follows.

Step 1: Input noisy image

Step 2: Decomposition of the image through wavelet

Step 3: Threshold the wavelet coefficients for noise removal

Step 4: Reconstruction of denoised image through inverse wavelet operations The flowchart of our proposed method is given in Fig 3.

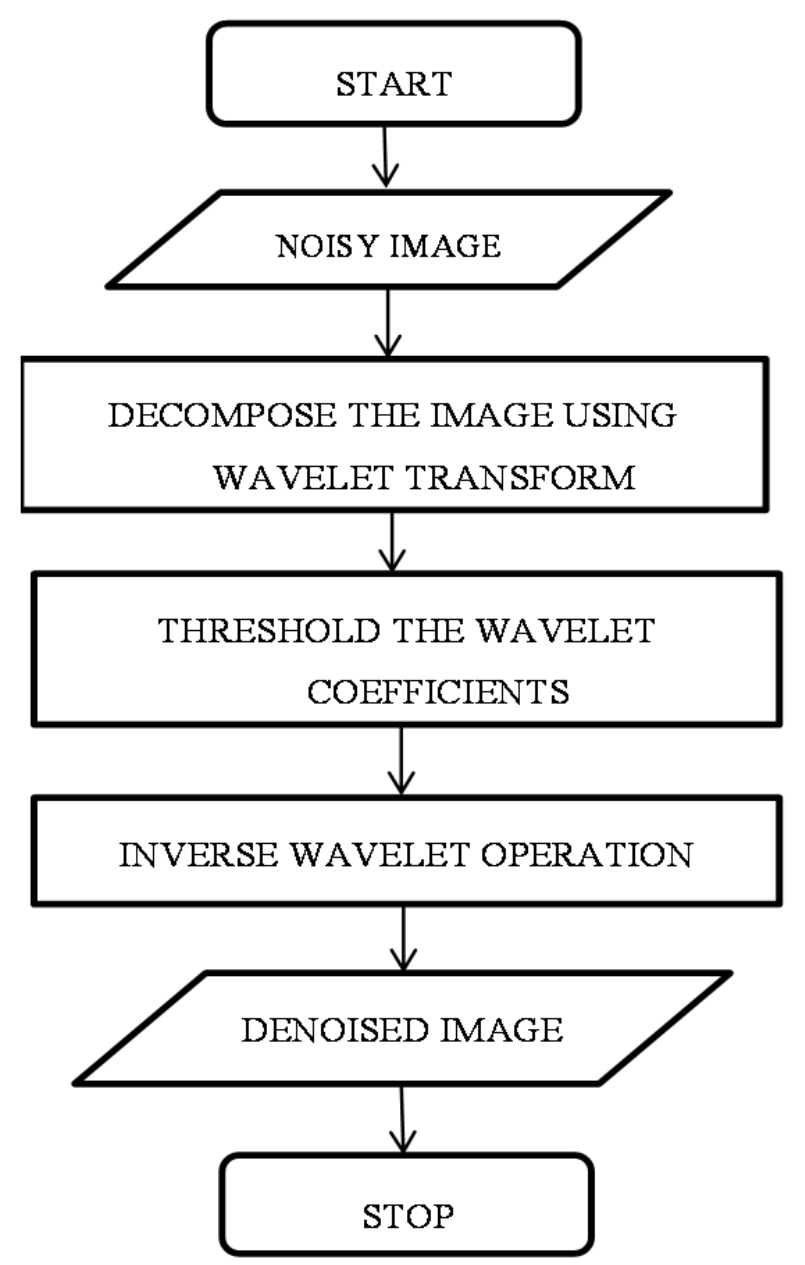

Fig3. Flowchart of Our Proposed Method

The detailed implementation of above mentioned steps are as follows.

Step1: Input noisy image

To evaluate our proposed method, the ultrasound images from various internet sources are selected to test our algorithm. The normal ultrasound images are subject to add the speckle noise with given noise level. Then, the noisy ultrasound image is put as an input to our proposed algorithm.

Step 2: Decomposition of the image through wavelet

As the mode of decomposition structures are discussed in above section, the DWT decompose the approximation sub-band in successive levels but the all other detail sub-bands are also decomposed further in WPT. All the obtained wavelet coefficients are taken for further processes.

Step 3: Threshold the wavelet coefficients 
The thresholding part of this algorithm is a major part of the denoising process. We have experimented our methods with different thresholding values. The selection of the threshold value is taken by around the value of universal threshold. We implement both the hard and soft thresholding on the wavelet coefficients and those results are taken into account for further reconstruction process. In addition, we keep the approximation coefficients as it as which means that the detail coefficients only subject to threshold.

Step 4: Reconstruction of denoised image through inverse wavelet operations

The inverse operations like inverse Discrete Wavelet Transform and inverse Wavelet Packet Transform are applied to the thresholded wavelet coefficients to get the denoised image.

\section{B. Evaluation Metrics}

To assess the quality of the denoised images, the evaluation metric called Peak Signal to Noise Ratio (PSNR) is used. The formula for PSNR is defined as follows.

$$
P S N R=10 \cdot \log _{10}\left(\frac{\operatorname{Max}_{i}^{2}}{M S E}\right)(1)
$$

where, $\operatorname{Max}_{i}$ is maximum intensity in an image, and $M S E$ is defined as

$$
M S E=\frac{1}{M N} \sum_{y=1}^{M} \sum_{x=1}^{N}\left[\operatorname{Im}(x, y)-\operatorname{Im}^{\prime}(x, y)\right]^{2}(2)
$$

where, $\operatorname{Im}(\mathrm{x}, \mathrm{y})$ is the original image, $\operatorname{Im}^{\prime}(\mathrm{x}, \mathrm{y})$ is the denoised image and $M, N$ is the dimensions of the images.

\section{Results and Discussion}

As mentioned earlier, a noisy ultrasound image is decomposed with the wavelet transform (here we used DWT and WPT). There are number of wavelet families are existing such as Haar, Daubechies, Symlets, Coiflets, Biorthogonal wavelets, Reverse biorthogonal wavelets, Morlets and so on. The selected wavelets are used to decompose the image in DWT and WPT. The Table.I shows that the results of our proposed method with the threshold value of fifty for an ultrasound image that is include as a first image of the fig 4.

Table I. Results for different selected wavelet family

\begin{tabular}{|c|c|c|c|c|}
\hline \multirow{2}{*}{ Wavelet } & \multicolumn{4}{|c|}{ PSNR (db) } \\
\cline { 2 - 5 } & \multicolumn{2}{|c|}{ DWT } & \multicolumn{2}{c|}{ WPT } \\
\cline { 2 - 5 } & Hard & Soft & 30.8941 & 28.7777 \\
\hline Haar & 30.6487 & 28.6423 & 33.5259 & 31.2451 \\
\hline $\mathrm{db} 4$ & 33.5759 & 31.2298 & 33.8230 & 31.5034 \\
\hline sym9 & 33.7627 & 33.7765 & 34.6503 & 33.4755 \\
\hline coif5 & 33.8364 & 31.6037 & 34.8438 & 33.5626 \\
\hline bior6.8 & 34.1719 & 31.6291 & 34.3575 & 33.0729 \\
\hline rbio5.5 & 34.3806 & 33.1056 & & \\
\hline
\end{tabular}

From our analysis, it is evident from Table. I that biorthogonal wavelet (bior6.8) given good PSNR values than others. For the further results taken for different threshold values, we have used this biorthogonal wavelet.

The resultant images of our proposed algorithms are given in Fig 4. A threshold level of fifty is set to threshold the wavelet coefficients in order to denoise the given ultrasound image. In Fig.4, the Column 1 contains the original tested image, column 2 contains the speckle noise added image, and the denoised images using DWT are given in column 3 and the denoised images using WPT is given in column 4. 

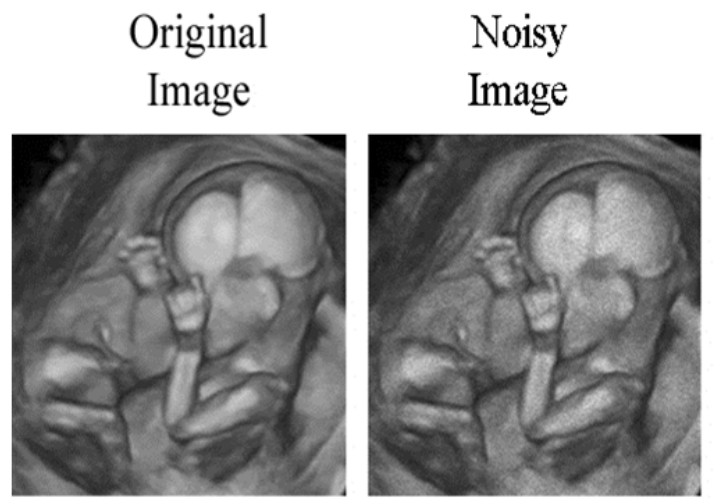

Denoised Image

(DWT)
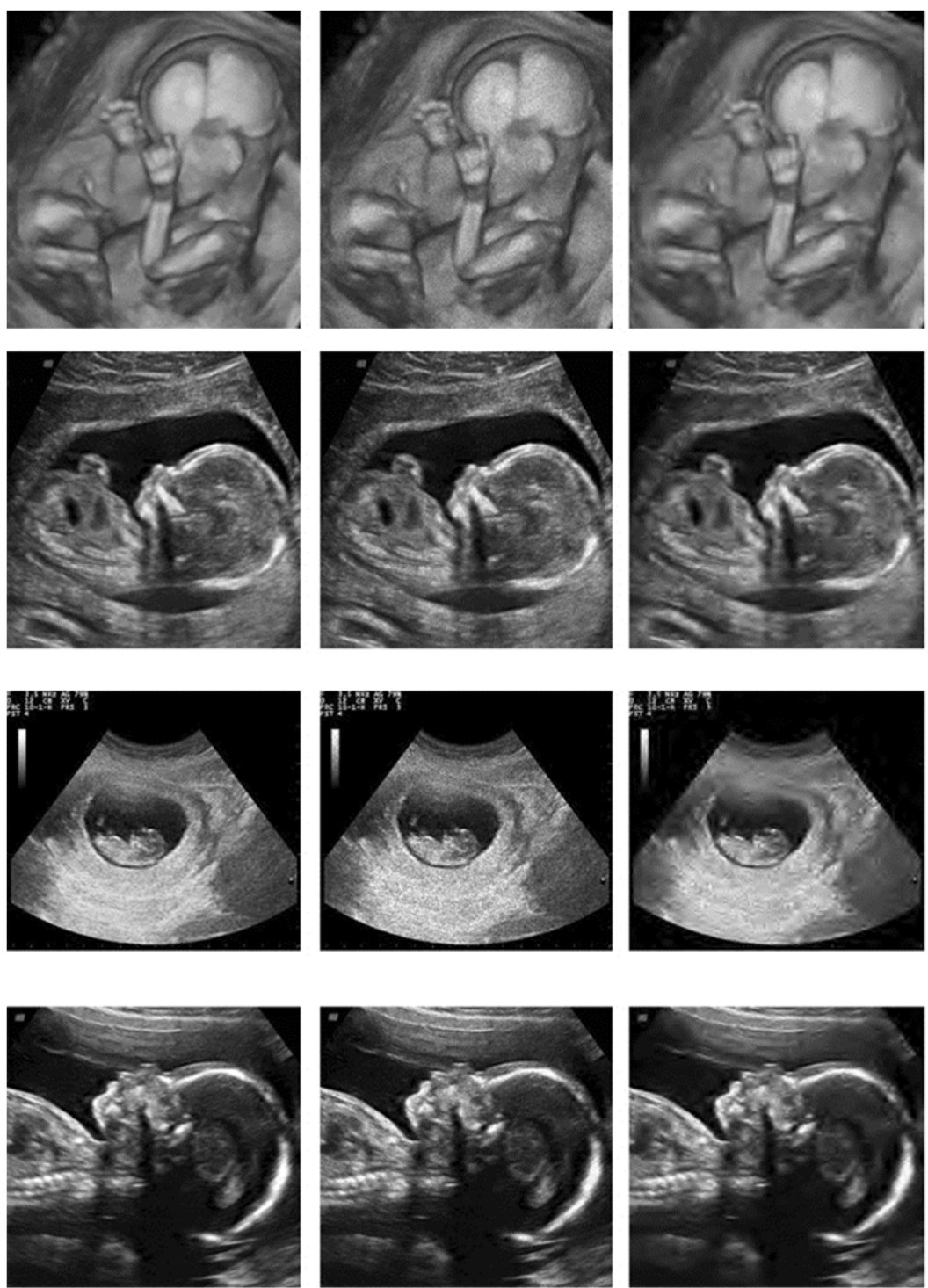

Fig 4. Resultant images of our proposed method
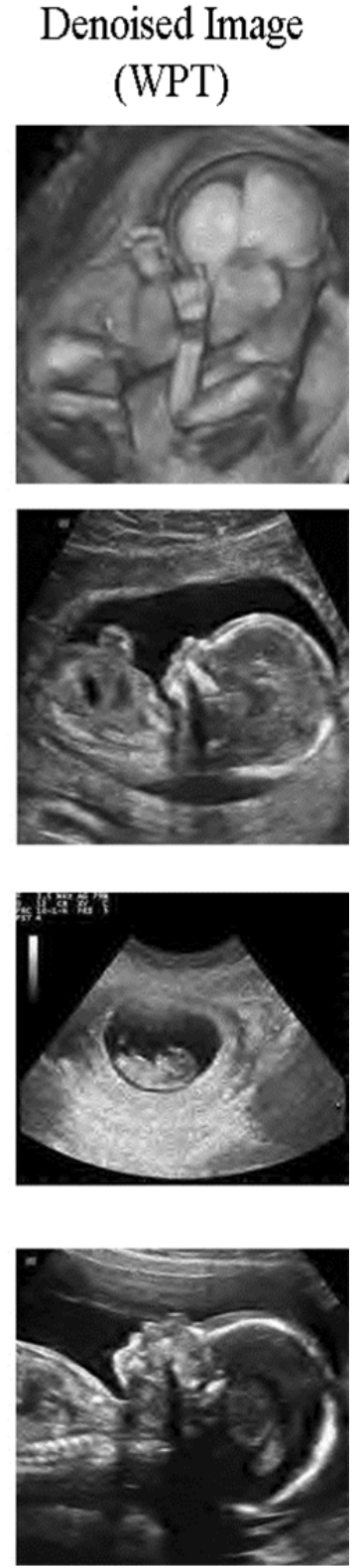

The numeric results for our proposed method in terms of PSNR for different threshold values are given in Table. II. It shows that hard thresholding of wavelet coefficients gives better results than the soft thresholding technique. Moreover, it is established that using of Wavelet Packet Transform for decomposing those images yields a comparable small scaled result than Discrete Wavelet Transform. 
Table II. Results for different threshold values

\begin{tabular}{|c|c|c|c|c|c|}
\hline \multirow{3}{*}{ Image } & \multirow{3}{*}{ Threshold value } & \multicolumn{4}{|c|}{ PSNR (db) } \\
\hline & & \multicolumn{2}{|c|}{ DWT } & \multicolumn{2}{|c|}{ WPT } \\
\hline & & Hard & Soft & Hard & Soft \\
\hline \multirow{4}{*}{ Image 1} & 50 & 34.1719 & 31.6291 & 34.8438 & 33.5626 \\
\hline & 55 & 33.9390 & 31.2040 & 34.6637 & 33.2206 \\
\hline & 60 & 33.6697 & 30.8679 & 33.6780 & 30.8957 \\
\hline & 80 & 32.2168 & 29.6898 & 32.3351 & 29.7163 \\
\hline & & & & & \\
\hline \multirow{4}{*}{ Image 2} & 50 & 30.1974 & 27.7343 & 30.3965 & 28.4895 \\
\hline & 55 & 29.7528 & 27.3675 & 30.0630 & 28.2086 \\
\hline & 60 & 29.3944 & 27.0262 & 29.6529 & 27.9485 \\
\hline & 80 & 28.2186 & 26.0070 & 28.2074 & 26.0245 \\
\hline \multirow{4}{*}{ Image 3} & 50 & 27.4448 & 25.4626 & 27.9249 & 26.8004 \\
\hline & 55 & 27.1234 & 25.1673 & 27.3221 & 25.5455 \\
\hline & 60 & 26.8336 & 24.8636 & 27.0086 & 25.2738 \\
\hline & 80 & 25.7625 & 23.9166 & 26.0374 & 24.4498 \\
\hline \multirow{4}{*}{ Image 4} & 50 & 30.1751 & 27.7712 & 30.3598 & 28.5635 \\
\hline & 55 & 29.8350 & 27.4155 & 29.8884 & 27.4069 \\
\hline & 60 & 29.5136 & 27.0487 & 29.5212 & 27.0619 \\
\hline & 80 & 28.3352 & 26.0147 & 28.3636 & 25.9666 \\
\hline
\end{tabular}

\section{CONCLUSIONS}

In this paper, we proposed a frequency domain noise removal technique that uses wavelet transforms. Particularly, this despeckling algorithm tested with ultrasound images with two different decomposing strategies such as DWT and WPT. By having the results obtained with our proposed method we can conclude that WPT decomposition structure gives little more better results than DWT. DWT sometimes give good results than WPT but it is evident from our obtained results that the WPT dominantly gives better results over DWT. For the type of ultrasound images, a wavelet based denoising method yields good performance particularly on the hard thresholding.

\section{REFERENCES}

[1] V. S. Prasath and P. Kalavathi "Mixed Noise Removal Using Hybrid Fourth Order Mean Curvature Motion". Advances in Signal Processing and Intelligent Recognition Systems, pp. 625-632, Springer International Publishing, 2016.

[2] P. Kalavathi, M. Abinaya and S. Boopathiraja, "Removal of Speckle Noise in Ultrasound Images using Spatial Filters", National Conference on Computational Methods, Communication Techniques and Informatics, pp.174-177, 2017. (ISBN: 978-81-933316-1-3)

[3] www.radiologyinfo.org.

[4] F. Benzarti and H. Amiri, "Speckle noise reduction in medical ultrasound images", arXiv preprint arXiv: 1305.1344, 2013.

[5] P. Kalavathi and T. Priya, "Removal of Impulse Noise Using Histogram-Based Localized Wiener Filter for MR Brain Image Restoration", IEEE Digital Explorer, 2016.

[6] J. W. Goodman, "Some fundamental properties of speckle," Journal of Optical Society of America (JOSA), vol. 66, no. 11, pp. 1145$1149,1976$.

[7] P. Kalavathi and P. Sowdeeswari, "Edge Enhanced Anisodiffusion Filter for Denoising Gaussian Noise in Medical Images", International Journal of Control Theory and Applications, vol. 9 no. 27, pp. 203-210, 2016.

[8] K. S. Raju, M.S Nasir, and T. M. Devi, "Filtering Techniques to reduce Speckle Noise and Image Quality Enhancement methods on Satellite Images", IOSR Journal of Computer Engineering (IOSR-JCE), pp.2278-0661, 2013.

[9] S.G. Mallat, "A Theory for Multiresolution Signal Decomposition: The Wavelet Representation", IEEE transactions on pattern analysis and machine intelligence, Vol. 11, No.7, PP. 674-693, 1989.

[10] I. Daubechies, "Ten lectures on wavelets", SIAM, New York, 1992.

[11] R. X. Gao, R. Yan, "Wavelets - Theory and Applications for Manufacturing", Signal \&Communication, Springer, ISBN 978-1-4419$1545-0$.

[12] MATLAB R2017a Documentation, www.mathworks.com 


\section{AUTHOR PROFILE}

P. Kalavathi Associate Professor and Head in the Department of Computer Science and Applications, Gandhigram Rural Institute-Deemed University, obtained her BCA degree from Mother Teresa Women's University, Kodaikanal; Post-Graduate Degree in Computer Applications (MCA) in GRI-DU; M. Phil in Computer Science from Bharathidasan University, Trichirappalli; and Doctoral Degree in Computer Science and Applications from GRI-DU, Gandhigram. She Qualified UGC-NET for Lectureship in 2000. She has 17 years of Teaching and 10 years of Research Experience. The Author has more than 50 research articles in various journals and edited volumes. Her research area focuses on Digital Image Processing, Medical image Segmentation \& Analysis and Medical Image Compression. She is also serving as a reviewer of many international Conferences and various journals in IEEE, Springer, Elsevier etc. The author is Life Time Member of Indian Society for Technical Education (ISTE), New Delhi.

S. Boopathiraja Research Scholar in Department of Computer Science and Applications, Gandhigram Rural Institute-Deemed University, obtained his Under-Graduate degree in Mathematics from GRI-DU; PostGraduate Degree in Computer Applications (MCA) from Institute of Road and Transport Technology (IRTT), Erode; M. Phil in Computer Science from GRI-DU; He has two years of Research Experience and his research area focuses on Medical Image Analysis and Digital Image Compression.

M. Abinaya Pursuing her M. Phil Degree in Computer Science from Department of Computer Science and Applications, Gandhigram Rural Institute-Deemed University, Gandhigram. 